\title{
Dichlorphenamide efficacy in the primary periodic paralyses
}

\begin{abstract}
Introduction: The periodic paralyses are rare genetic neuromuscular disorders that cause significant morbidity with episodic symptoms of muscle paralysis lasting from hours to weeks. There are no consensus treatment guidelines. Current treatment options include diuretics and the carbonic anhydrase inhibitor acetazolamide, but only anecdotal evidence and case reports support their use.
\end{abstract}

Areas covered: We consider the evidence for the carbonic anhydrase inhibitor dichlorphenamide. This is the only treatment for periodic paralysis to have undergone randomised controlled trials. Although there has never been a comparative trial of dichlorphenamide and acetazolamide, many patients anecdotally report greater benefit from dichlorphenamide. However, it has been unavailable worldwide since 2011 until its recent reintroduction to the market in late 2015.

Expert opinion: There is level I evidence for the efficacy of dichlorphenamide in periodic paralysis. The ubiquitous nature of carbonic anhydrase enzymes mean that systemic administration of a carbonic anhydrase inhibitor leads to common dose-dependent side effects. In hypokalaemic periodic paralysis the benefits seem to outweigh these with acceptable tolerability. A successful consequence of recent randomised controlled trials was the re-introduction of dichlorphenamide to the commercial market following FDA approval in August 2015. Orphan status of dichlorphenamide for the treatment of periodic paralysis is to be welcomed but the current pricing is potentially prohibitive.

\section{Keywords}

Keywords: Andersen-Tawil syndrome, Carbonic anhydrase inhibitors, Hyperkalaemic periodic paralysis, Hypokalaemic periodic paralysis, Periodic Paralyses, Sulphonamides 


\section{Introduction}

The primary periodic paralyses are rare genetic neuromuscular disorders. They include hypokalaemic periodic paralysis (hypoPP), hyperkalaemic periodic paralysis (hyperPP) and Andersen-Tawil Syndrome (ATS). HypoPP and hyperPP are disorders that only affect skeletal muscle ${ }^{1}$. ATS is characterised by a triad of periodic muscle paralysis, dysmorphic features and cardiac conduction abnormalities ${ }^{2,3}$.

Paroxysmal attacks of skeletal muscle weakness or paralysis accompanied by high or low levels of serum potassium are the defining clinical feature of the periodic paralyses. They usually present in the first or second decade and attacks of muscle weakness typically follow a period of immobility, especially after exercise. Other provoking factors are more specific to the sub-type of paralysis e.g. attacks in hypoPP are precipitated by large carbohydrate meals which stimulate insulin release and ultimately hypokalaemia ${ }^{1}$. Limb and trunk muscles are commonly affected, although any skeletal muscle including for example respiratory muscles may be involved ${ }^{4}$. Attacks of muscle weakness last typically from minutes to days (although can be weeks) depending on the sub-type and are extremely debilitating. The altered levels of serum potassium that accompany the attacks can induce cardiac arrhythmia ${ }^{5}$. In ATS cardiac conduction abnormalities and potentially fatal ventricular arrhythmias can occur independently of the muscle symptoms ${ }^{6,7}$.

The attacks of muscle paralysis are due to dysfunction of voltage gated ion channels which reside in the membrane of skeletal muscles ${ }^{8}$. These channels regulate muscle membrane excitability and control muscle contraction and relaxation. Genetic mutation of the genes coding for these ion channels intermittently causes prolonged depolarisation of the muscle membrane rendering the muscle inexcitable and unable to contract ${ }^{8}$. In hypoPP this is produced by an aberrant inward cation current that is created by arginine mutations of the 
sarcolemmal sodium or calcium channel voltage sensors ${ }^{9}$. In hyperPP there is persistent sodium current due to impaired sodium channel inactivation and in ATS there is reduced potassium conductance. All three mechanisms are unified by destabilising the muscle membrane and leading to prolonged depolarisation. ${ }^{10}$

The periodic paralyses are rare, the estimated point prevalence in England is: hyperkalemic periodic paralysis $0.17 / 100,000$, hypokalemic periodic paralysis $0.13 / 100,000$, and AndersenTawil syndrome (ATS) 0.08/100,000 ${ }^{11}$.

Obtaining evidence of treatment efficacy in this group of disorders has partly been hampered by their rarity, as this impedes large adequately powered clinical trials. There are no recognised consensus treatment guidelines ${ }^{12}$.

\section{Overview of the market}

\subsection{What are the unmet needs of currently available therapies?}

Treatment options include potassium sparing or wasting diuretics to maintain a normal serum potassium level as prophylactic treatment. In the case of hypoPP potassium supplements at the onset of an attack can be used to abort or reduce symptom duration and severity. However all of these options rely on case report or small series reporting of efficacy ${ }^{12}$. The majority of published data relates to the use of carbonic anhydrase inhibitors, acetazolamide and dichlorphenamide that have been used as prophylactic agents in all the periodic paralyses ${ }^{13-15}$

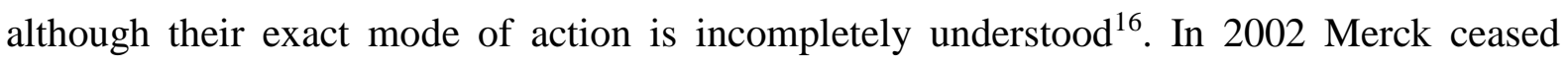
production of dichlorphenamide and it has been unavailable worldwide since 2011. Acetazolamide remains widely available and affordable although is not licensed specifically for the treatment of periodic paralysis. There is no randomised controlled trial evidence supporting its use. However while it clearly has benefit across the periodic paralyses one retrospective series suggested up to $40-50 \%$ of patients with hypoPP are unresponsive to acetazolamide $^{17}$. It is also our clinical experience from the UK National referral centre for 
skeletal muscle channelopathies that a significant proportion of patients with PP respond significantly better to dichlorphenamide than acetazolamide.

\subsection{Which competitor compounds/classes of compounds are in the clinic/late}

\section{development?}

Activation of a cotransporter of sodium-potassium-and two chloride ions (NKCC) promotes chloride accumulation within muscle cells. Intramuscular accumulation of chloride contributes to the propensity of the muscle membrane to become depolarised (and muscle paralysis to occur) in a low potassium environment ${ }^{18}$. Bumetanide is a diuretic that inhibits this cotransporter. Based on the hypothesis that inhibiting intramuscular chloride accumulation would reduce or prevent muscle membrane depolarisation and muscle paralysis in hypoPP two studies examined the in vitro and in vivo effects of bumetanide using a murine mode $^{19,20}$. In both scenarios bumetanide was effective in aborting an episode of muscle weakness in the presence of hypokalaemia and in preventing muscle weakness from occurring if given prior to the induction of hypokalaemia. In the in vivo murine model its benefit in recovering or sustaining muscle strength was similar to acetazolamide in individual cases, although a greater number of animals in total responded to bumetanide than acetazolamide ${ }^{20}$.

As a diuretic, bumetanide can also produce hypokalaemia. Whether this would be clinically relevant in hypoPP is unknown. A clinical safety study is currently open using intravenous bumetanide versus placebo in hypoPP. The half-life of bumetanide however is very short, approximately one and a half hours which will inhibit its use as a daily prophylactic. It may however have potential to be used as a prophylactic agent if taken just prior to activities known to provoke an attack of paralysis e.g. before sport. 


\section{Introduction to dichlorphenamide}

The carbonic anhydrase inhibitor acetazolamide was initially given to patients with hyperkalaemic periodic paralysis in which attacks of paralysis occur in conjunction with raised serum potassium levels because of its ability to increase urinary potassium excretion ${ }^{13}$. Despite seeming to be an unlikely candidate for treatment of hypokalaemic periodic paralysis, a prophylactic benefit was subsequently also reported in this disorder ${ }^{21,22}$. Experimental work has supported a rationale for carbonic anhydrase inhibitors due to their effect on $\mathrm{pH}^{23}$, improved sarcolemmal conductance of potassium via calcium activated potassium channels ${ }^{24,25}$ and observation of a reduced number of vacuoles seen in the muscle biopsies of potassium depleted rats administered acetazolamide ${ }^{26}$. Acetazolamide and dichlorphenamide have been the mainstay of treatment for the periodic paralyses for the last half a century.

\section{Chemistry}

Carbonic anhydrases are ubiquitously expressed isoenzymes that catalyse the reversible conversion of water and carbon dioxide to protons and bicarbonate with corresponding effects on $\mathrm{pH}$ balance. This reaction is key to numerous physiological processes including respiration, $\mathrm{pH}$ and $\mathrm{CO} 2$ homeostasis; electrolyte secretion, biosynthetic reactions e.g. gluconeogenesis, lipogenesis and ureagenesis, bone resorption and calcification ${ }^{27}$. Dichlorphenamide (DCP), a benzenedisulfonamide, is a carbonic anhydrase inhibitor (CAI) ${ }^{28}$.

\section{Pharmacodynamics, pharmacokinetics and metabolism}

Carbonic anhydrase inhibitors have a weak diuretic action ${ }^{29}$. They act on the proximal tubule of the kidney to inhibit bicarbonate resorption. Consequently there is increased bicarbonate, sodium and potassium excretion in the urine which causes an accompanying diuresis and metabolic acidosis. Although they have been used in the management of hypertension and 
congestive cardiac failure, other more potent diuretics have largely superseded their use other than as additive agents.

One of their main therapeutic roles has been in the treatment of glaucoma ${ }^{30}$. The major constituent of aqueous humour is sodium bicarbonate. Carbonic anhydrase inhibitors reduce the excretion of this with a resultant drop in intra-ocular pressure.

The ability of CAIs to inhibit carbonic anhydrases have since been manipulated for numerous other medical conditions including epilepsy, high altitude sickness, raised intracranial pressure, episodic ataxia, myotonic disorders ${ }^{31}$ and periodic paralysis. More recent research has suggested they could be developed in newer fields including osteoporosis, obesity and oncology 27 .

Systemic CAIs are well absorbed via the GI tract and excreted unchanged by the kidneys. Dose adjustments may therefore need to be made for impaired creatinine clearance. A single oral dose has benefit for approximately 8 to 12 hours. Carbonic anyhdrases are ubiquitously expressed $^{32}$, and systemic administration of a CAI leads to unselected CA enzyme blockade. There are multiple isoforms of carbonic anhydrase and each carbonic anhydrase inhibitor has a variable affinity for each isoform ${ }^{28}$.

Sulphonamides include drugs with both antibacterial and non-antibacterial properties, DCP and acetazolamide belonging to the latter group. Allergic reactions to sulphonamide antibiotics are relatively common and there is often concern over cross-reactivity when using CAIs $^{33}$. However the antibiotic sulphonamides contain an arylamine group (an amine attached to a benzene ring- see figure 1) which is thought to be implicated in the majority of allergic reactions. DCP and other CAIs lack this moiety and they have been used safely in those with known sulphonamide antibiotic sensitivity but caution is still advised especially in cases of severe allergy ${ }^{34}$. 


\section{Clinical efficacy}

The initial drug safety profile of DCP was ascertained from studies in its primary indication, the treatment of glaucoma. Dose efficacy and ascertainment of any disease specific side effects in periodic paralysis were indicated from non-randomised and often small scale studies although more recent phase III studies have now added to this body of knowledge.

\subsection{Phase III trials}

In 2000 a randomised double blind placebo controlled trial was reported which attempted to address the question of DCP efficacy and tolerability when used in the treatment of periodic paralysis $^{35}$. In a seven site study, DCP was compared to placebo in two groups of patients, those with a diagnosis of hypoPP and those classed as potassium sensitive periodic paralysis by the investigators. This latter group encompassed hyperkalaemic periodic paralysis and paramyotonia congenita with episodes of muscle paralysis. Diagnosis was largely based on clinical criteria and a genetic mutation was not essential for inclusion. Trial design consisted of an eight week baseline assessment of attack frequency and severity followed by two cross over nine week treatment phases (DCP or placebo) separated by a nine week washout phase. Forty two patients with hypoPP were enrolled. Thirty four completed the study. Two dropouts were due to inability to tolerate adverse events of DCP (dizziness and difficulty concentrating at work) and the others lost to follow up or requested withdrawal for other reasons. Based on those who completed the trial the primary end point of intolerable increase in attack frequency or severity was reached in fifteen, two reaching the end point in both treatment arms (DCP and placebo) and thirteen exhibiting a preference. For those exhibiting a preference eleven were taking placebo and two taking DCP. Secondary endpoints of frequency and severity of attacks were also significantly lower in the DCP treated group. 
Thirty one patients with PSPP were enrolled, twenty four completing both treatment arms of the study. Two of the dropouts were due to adverse events whilst taking DCP (rash and memory loss). Of those who completed the study a significantly greater reduction in attack frequency (the primary outcome for this group) and severity was demonstrated with DCP compared to placebo.

This trial was the first to provide randomised placebo controlled evidence for the use of DCP in the periodic paralyses and supported clinical impression of efficacy.

A second randomised placebo controlled trial with a one year open label extension phase attempting to ascertain if benefit was maintained in the longer-term was reported in $2016^{36}$. This twelve centre international study randomised participants with a diagnosis of hypoPP or hyperPP between 2007 and 2013. Diagnosis again encompassed a clinically based criteria and genetic diagnosis was not essential but $75 \%$ of hypoPP and $67 \%$ of hyperPP participants did have a genetic diagnosis made. Participants were randomised to an initial 9 week blinded treatment phase with either DCP or placebo followed by one year of continued treatment with DCP. The original trial design had included randomisation to either DCP or acetazolamide. However, this subsequently had to be dropped, as a significant proportion of patients report DCP to be more effective than acetazolamide and were reluctant to participate when there was a chance of being randomised to acetazolamide. Forty four hypoPP and twenty one hyperPP participants were randomised to the double blind phase. Five hypoPP participants receiving placebo, but none receiving DCP were moved prematurely before the end of the blinded nine week treatment phase to the open label DCP phase due to acute worsening of symptoms. This was mirrored in the hyperPP arm, with two receiving placebo, but none receiving DCP moving early into the open label phase.

In the hypoPP group there were statistically significant improvements in attack rate, severity and duration with DCP during the double-blind nine week treatment phase. There were also 
improvements in quality of life demonstrated by the physical component score of the SF-36. Improvement in attack frequency also seemed to persist during the extension phase. In the hyperPP group there was a significant improvement in attack severity but not rate of attacks or attack duration. No changes were seen in quality of life. Despite an admirable attempt by the authors to recruit subjects with a very rare disease into this trial they did not ultimately meet their recruitment targets particularly for hyperPP and this may have affected the outcomes.

\subsection{Post-marketing surveillance}

Following the conclusion of this international study DCP was granted Food and Drug Administration approval in August 2015. Taro pharmaceuticals began commercial manufacture in the USA but uptake in clinical practice has been slow due to expense and there are no post-marketing surveillance studies currently available.

\section{Safety and tolerability}

Carbonic anhydrase inhibitors can interact with other common medications e.g. non-steroidal anti-inflammatory drugs and anti-epileptics to potentially cause a severe metabolic acidosis or enhance drug toxicity. There is also a risk of severe acidosis in those with renal, liver or respiratory disease. A careful medical and drug history is therefore required before initiating these medications ${ }^{37}$.

Common dose dependent adverse effects of DCP include numbness and tingling of the extremities, metallic taste, fatigue, loss of libido, anorexia, headache, transient myopia and renal calculi. Short-term use in humans indicates that these adverse effects are minimal for single doses of less than $200 \mathrm{mg}^{38}$. In glaucoma studies DCP was less well tolerated than $\mathrm{ACZ}$ which likely reflects its greater potency as a $\mathrm{CAI}^{39}$. In general in ophthalmic medicine 
the side effects of systemic CAIs were rather intolerable and topical preparations developed. However the dosage of DCP used in glaucoma, $50 \mathrm{mg}$ every 6 hours, was twice the average dosage used in studies demonstrating the efficacy of DCP for the treatment of $\mathrm{PP}^{35,36}$. In the 2016 study of DCP in periodic paralysis $18 \%$ of participants taking DCP withdrew due to adverse events, the majority in the long-term extension phase ${ }^{36}$. Although paraesthesia was the most common side effect occurring in $47 \%$ of those taking DCP it was never the cause of withdrawal. Cognitive disorder was the second most commonly noted adverse event (19\%) and prominent enough that the authors recommended all patients being offered DCP should be counselled about the possibility, especially those in mentally demanding occupations. The development of renal calculi is often a concern when using long-term CAIs. Of the 53 participants (39 hypoPP, 14 hyperPP) who underwent renal tract assessment at the end of the trial eight developed new renal calculi (7 hypoPP, 1hyperPP) and two hypoPP participants had an increase in size of pre-existing calculi ${ }^{36}$. One participant withdrew because of painful calculi but it is unclear if the others were symptomatic or if treatment was necessary. However this indicates that monitoring of the renal tract for calculi in those on long-term DCP will be essential.

\section{Regulatory affairs}

Dichlorphenamide was originally marketed by Merck \& CO., INC., 1985 NDC \#51672-4144 for the treatment of glaucoma but Merck ceased manufacture in 2002. We are not aware of any adverse regulatory actions that have been taken against Dichlorphenamide in any country and the FDA DOCID: fr06au07-57 asserts that it was not withdrawn from sale for reasons of 'safety or effectiveness'. Following the success of the international trials of DCP, it was granted orphan drug status for periodic paralysis by the FDA in the USA and commercial 
manufacture began by Taro pharmaceuticals. The price was approximately $\$ 100000$ per patient per year. Uptake was very low and in May 2016 Taro announced it was stopping commercial production in the USA. An application for a European marketing authorisation is currently being considered although the final price in Europe is yet to be established.

\section{Conclusion}

There is level I evidence for the efficacy of dichlorphenamide in the treatment of hypoPP and hyperPP. There have been no randomised controlled trials in Andersen-Tawil syndrome but case reports support benefit and the clinical symptom of periodic paralysis mirrors the other two conditions. Although there have been no head to head studies with acetazolamide, many patients report a much better response to DCP in clinic. It is unknown if side effects are also comparatively more troublesome although the incidence was relatively high in one randomised controlled trial. However benefit did seem to outweigh adverse events in the hypoPP arm of that study.

\section{Expert opinion}

The randomised trial evidence supports the clinical impression gained over many years; DCP is an effective treatment for periodic paralysis. Adverse events are relatively common but for the majority tolerable. Currently, a significant proportion of patients with periodic paralysis do not have adequate control of their symptoms from available treatments and suffer unnecessary morbidity as DCP is not available to them. The cost of dichlorphenamide when marketed as a treatment for glaucoma was approximately $£ 100$ for 100 tablets. It was a huge success for the rare disease community to be able to conduct randomised controlled trials in such rare disease. Orphan status of dichlorphenamide for the treatment of periodic paralysis was celebrated but the ensuing price rise of DCP to $\$ 100000$ per patient per year when 
brought back to the commercial market has caused significant affordability issues for healthcare providers. Hopefully if EMA approval is obtained and DCP launched in Europe it will be affordable to the health service.

\section{Drug Summary Box}

Drug name: dichlorphenamide

Phase: commercially manufactured USA August 2015 (ceased May 2016). Application for EMA pending.

Indication: Hypokalaemic periodic paralysis, Hyperkalaemic periodic paralysis and Andersen-Tawil Syndrome

Pharmacology description/mechanism of action: Carbonic anhydrase inhibitor

Route of administration: Oral

Pivotal trial(s):

Tawil R, McDermott MP, Brown R, Jr., et al. Randomized trials of dichlorphenamide in the periodic paralyses. Working Group on Periodic Paralysis. Ann Neurol 2000;47:46-53

Sansone VA, Burge J, McDermott MP, et al. Randomized, placebo-controlled trials of dichlorphenamide in periodic paralysis. Neurology 2016;86:1408-16 
Reference List

1. Venance SL, Cannon SC, Fialho D, et al. The primary periodic paralyses: diagnosis, pathogenesis and treatment. Brain 2006;129:8-17: published online , doi:awh639 [pii];10.1093/brain/awh639 [doi]

2. Andersen ED, Krasilnikoff PA, Overvad H. Intermittent muscular weakness, extrasystoles, and multiple developmental anomalies. A new syndrome? Acta Paediatr Scand 1971;60:55964

3. Tawil R, Ptacek $L$, Pavlakis SG, et al. Andersen's syndrome: potassium-sensitive periodic paralysis, ventricular ectopy, and dysmorphic features. Ann Neurol 1994;35:326-30: published online , doi:10.1002/ana.410350313 [doi]

4. Kil TH, Kim JB. Severe respiratory phenotype caused by a de novo Arg528Gly mutation in the CACNA1S gene in a patient with hypokalemic periodic paralysis. Eur J Paediatr Neurol 2010;14:278-81: published online , doi:S1090-3798(09)00156-1 [pii];10.1016/j.ejpn.2009.08.004 [doi]

5. Kim JB, Lee KY, Hur JK. A Korean family of hypokalemic periodic paralysis with mutation in a voltage-gated calcium channel (R1239G). J Korean Med Sci 2005;20:162-5: published online , doi:200502162 [pii];10.3346/jkms.2005.20.1.162 [doi]

6. Zhang L, Benson DW, Tristani-Firouzi M, et al. Electrocardiographic features in AndersenTawil syndrome patients with KCNJ2 mutations: characteristic T-U-wave patterns predict the KCNJ2 genotype. Circulation 2005;111:2720-6: published online , doi:CIRCULATIONAHA.104.472498 [pii];10.1161/CIRCULATIONAHA.104.472498 [doi]

7. Kostera-Pruszczyk A, Potulska-Chromik A, Pruszczyk $P$, et al. Andersen-Tawil syndrome: report of 3 novel mutations and high risk of symptomatic cardiac involvement. Muscle Nerve 2015;51:192-6: published online, doi:10.1002/mus.24293 [doi]

8. Cannon SC. Pathomechanisms in channelopathies of skeletal muscle and brain. Annu Rev Neurosci 2006;29:387-415: published online, doi:10.1146/annurev.neuro.29.051605.112815 [doi]

9. Sokolov S, Scheuer T, Catterall WA. Gating pore current in an inherited ion channelopathy. Nature 2007;446:76-8: published online , doi:nature05598 [pii];10.1038/nature05598 [doi]

10. Cannon SC. Channelopathies of skeletal muscle excitability. Compr Physiol 2015;5:761-90: published online, doi:10.1002/cphy.c140062 [doi]

11. Horga A, Raja Rayan DL, Matthews E, et al. Prevalence study of genetically defined skeletal muscle channelopathies in England. Neurology 2013;80:1472-5: published online , doi:WNL.0b013e31828cf8d0 [pii];10.1212/WNL.0b013e31828cf8d0 [doi]

12. Sansone V, Meola G, Links TP, et al. Treatment for periodic paralysis. Cochrane Database Syst Rev 2008;CD005045: published online , doi:10.1002/14651858.CD005045.pub2 [doi]

13. McArdle B. ADYNAMIA EPISODICA HERED1TARIA AND ITS TREATMENT. Brain 1962;85:121-48 
14. Resnick JS, Engel WK, Griggs RC, Stam AC. Acetazolamide prophylaxis in hypokalemic periodic paralysis. N Engl J Med 1968;278:582-6: published online , doi:10.1056/NEJM196803142781102 [doi]

15. Junker J, Haverkamp W, Schulze-Bahr E, et al. Amiodarone and acetazolamide for the treatment of genetically confirmed severe Andersen syndrome. Neurology 2002;59:466

16. Matthews E, Hanna MG. Muscle channelopathies: does the predicted channel gating pore offer new treatment insights for hypokalaemic periodic paralysis? J Physiol 2010;588:187986: published online , doi:jphysiol.2009.186627 [pii];10.1113/jphysiol.2009.186627 [doi]

17. Matthews $E$, Portaro $S$, Ke $Q$, et al. Acetazolamide efficacy in hypokalemic periodic paralysis and the predictive role of genotype. Neurology 2011;77:1960-4: published online , doi:WNL.0b013e31823a0cb6 [pii];10.1212/WNL.0b013e31823a0cb6 [doi]

18. Geukes Foppen RJ, van Mil HG, van Heukelom JS. Effects of chloride transport on bistable behaviour of the membrane potential in mouse skeletal muscle. J Physiol 2002;542:181-91: published online , doi:PHY_13298 [pii]

19. Wu F, Mi W, Cannon SC. Bumetanide prevents transient decreases in muscle force in murine hypokalemic periodic paralysis. Neurology 2013;80:1110-6: published online , doi:WNL.0b013e3182886a0e [pii];10.1212/WNL.0b013e3182886a0e [doi]

20. Wu F, Mi W, Cannon SC. Beneficial effects of bumetanide in a CaV1.1-R528H mouse model of hypokalaemic periodic paralysis. Brain 2013;136:3766-74: published online , doi:awt280 [pii];10.1093/brain/awt280 [doi]

21. Resnick JS, Engel WK, Griggs RC, Stam AC. Acetazolamide prophylaxis in hypokalemic periodic paralysis. N Engl J Med 1968;278:582-6

22. Griggs RC, Engel WK, Resnick JS. Acetazolamide treatment of hypokalemic periodic paralysis. Prevention of attacks and improvement of persistent weakness. Ann Intern Med 1970;73:3948

23. Kuzmenkin A, Muncan V, Jurkat-Rott $K$, et al. Enhanced inactivation and $\mathrm{pH}$ sensitivity of $\mathrm{Na}(+)$ channel mutations causing hypokalaemic periodic paralysis type II. Brain 2002;125:835-43

24. Tricarico D, Barbieri M, Camerino DC. Acetazolamide opens the muscular KCa2+ channel: a novel mechanism of action that may explain the therapeutic effect of the drug in hypokalemic periodic paralysis. Ann Neurol 2000;48:304-12

25. Tricarico D, Barbieri $M$, Mele $A$, et al. Carbonic anhydrase inhibitors are specific openers of skeletal muscle BK channel of K+-deficient rats. FASEB J 2004;18:760-1

26. Tricarico $D$, Lovaglio $S$, Mele $A$, et al. Acetazolamide prevents vacuolar myopathy in skeletal muscle of $K(+)$-depleted rats. Br J Pharmacol 2008;154:183-90

27. Supuran CT. Carbonic anhydrases: novel therapeutic applications for inhibitors and activators. Nat Rev Drug Discov 2008;7:168-81: published online , doi:nrd2467 [pii];10.1038/nrd2467 [doi] 
28. Alterio V, De SG, Monti SM, et al. Carbonic anhydrase inhibitors: inhibition of human, bacterial, and archaeal isozymes with benzene-1,3-disulfonamides--solution and crystallographic studies. Bioorg Med Chem Lett 2007;17:4201-7: published online , doi:S0960-894X(07)00611-7 [pii];10.1016/j.bmcl.2007.05.045 [doi]

29. Carta F, Supuran CT. Diuretics with carbonic anhydrase inhibitory action: a patent and literature review (2. Expert Opin Ther Pat 2013;23:681-91: published online , doi:10.1517/13543776.2013.780598 [doi]

30. GONZALES-JIMENEZ E, LEOPOLD IH. Effect of dichlorphenamide on the intraocular pressure of humans. AMA Arch Ophthalmol 1958;60:427-36

31. Markhorst JM, Stunnenberg BC, Ginjaar IB, et al. Clinical experience with long-term acetazolamide treatment in children with nondystrophic myotonias: a three-case report. Pediatr Neurol 2014;51:537-41: published online , doi:S0887-8994(14)00331-2 [pii];10.1016/j.pediatrneurol.2014.05.027 [doi]

32. Supuran CT, Scozzafava A. Carbonic anhydrases as targets for medicinal chemistry. Bioorg Med Chem 2007;15:4336-50: published online , doi:S0968-0896(07)00337-9 [pii];10.1016/j.bmc.2007.04.020 [doi]

33. Johnson KK, Green DL, Rife JP, Limon L. Sulfonamide cross-reactivity: fact or fiction? Ann Pharmacother 2005;39:290-301: published online , doi:aph.1E350 [pii];10.1345/aph.1E350 [doi]

34. Platt $D$, Griggs RC. Use of acetazolamide in sulfonamide-allergic patients with neurologic channelopathies. Arch Neurol 2012;69:527-9: published online , doi:archneurol.2011.2723 [pii];10.1001/archneurol.2011.2723 [doi]

35. Tawil R, McDermott MP, Brown R, Jr., et al. Randomized trials of dichlorphenamide in the periodic paralyses. Working Group on Periodic Paralysis. Ann Neurol 2000;47:46-53

36. Sansone VA, Burge J, McDermott MP, et al. Randomized, placebo-controlled trials of dichlorphenamide in periodic paralysis. Neurology 2016;86:1408-16: published online , doi:WNL.0000000000002416 [pii];10.1212/WNL.0000000000002416 [doi]

37. Supuran CT. Drug interaction considerations in the therapeutic use of carbonic anhydrase inhibitors. Expert Opin Drug Metab Toxicol 2016;12:423-31: published online , doi:10.1517/17425255.2016.1154534 [doi]

38. ROCHELLE JB, III, MOYER JH, FORD RV. Dichlorphenamide as a diuretic agent. Am J Med Sci 1958;235:168-78

39. Lichter PR, Newman LP, Wheeler NC, Beall OV. Patient tolerance to carbonic anhydrase inhibitors. Am J Ophthalmol 1978;85:495-502 